\title{
Two-proton knockout on neutron-rich nuclei
}

D. Bazin ${ }^{\text {* }}$, B. A. Brown ${ }^{\text {ab }}$, C. M. Campbellab, J. A. Church ${ }^{\text {ab }}$, D. C. Dinca ${ }^{\text {ab }}$, J. Enders $^{\mathrm{a} \dagger}$, A. Gade ${ }^{\mathrm{ab}}$, T. Glasmacher ${ }^{\mathrm{ab}}$, P. G. Hansen ${ }^{\mathrm{ab}}$, W. F. Mueller ${ }^{\mathrm{a}}$, H. Olliver ${ }^{\mathrm{ab}}$, B. C. Perry ${ }^{\text {ab }}$, B. M. Sherrill ${ }^{\text {ab }}$, J. R. Terry ${ }^{\text {ab }}$ and J. A. Tostevin ${ }^{c}$

${ }^{a}$ National Superconducting Cyclotron Laboratory, Michigan State University, East Lansing, MI 48824, USA

${ }^{b}$ Department of Physics and Astronomy, Michigan State University, East Lansing, MI 48824, USA

${ }^{\mathrm{c}}$ Department of Physics, School of Physics and Chemistry, Guildford, Surrey GU2 7XH, United Kingdom

Two-proton knockout reactions on neutron-rich nuclei [1] have been studied in inverse kinematics at intermediate energy. Strong evidence that the two-proton removal from a neutron-rich system proceeds as a direct reaction is presented, together with a preliminary theoretical discussion of the partial cross sections based on eikonal reaction theory and the many-body shell model. They show that this reaction can be used to characterize the wave functions of the projectiles and holds great promise for the study of neutron-rich nuclei.

\section{Introduction}

A direct reaction proceeds from an initial to a final state of a system by the selective excitation of a small number of nucleonic degrees of freedom $[2,3]$. Its cross section is then specified by the wave functions describing these few particles. This has made direct reactions a powerful and widely applied tool for investigating one- and two-nucleon wave functions in nuclei. The present work extends the use of direct reactions by showing that two-proton removal from a neutron-rich system at high energy proceeds as a direct reaction. This is what would be expected intuitively from the asymmetry in proton and neutron separation energies which suppresses alternative reaction paths to the $-2 \mathrm{p}$ final state. To see this, consider the nucleus ${ }^{28} \mathrm{Mg}$ with a two-proton separation energy of $30.0 \mathrm{MeV}$ (see fig. 1), where an alternative to the direct path would be the formation of ${ }^{27} \mathrm{Na}$ in a one- proton knockout. Here, however, the channel for proton evaporation is closed up to the proton separation energy of $13.3 \mathrm{MeV}$ thus excluding the major part of the strength in this direct reaction. Even above the proton threshold, neutron evaporation (with a separation energy of $6.8 \mathrm{MeV}$ ) will be much more likely.

\footnotetext{
*E-mail address: bazin@nscl.msu.edu

${ }^{\dagger}$ present address: Institut für Kernphysik, TU Darmstadt, D-64289 Darmstadt, Germany

${ }^{\ddagger}$ present address: Constellation Technologies, Largo, FL 33777, USA
} 


\section{Experiment}

The total and differential cross sections for the formation of the two-proton knockout reaction residues from ${ }^{28} \mathrm{Mg},{ }^{30} \mathrm{Mg}$ and ${ }^{34} \mathrm{Si}$ on a ${ }^{9} \mathrm{Be}$ target were measured. The detection of coincident gamma rays provides partial cross sections, just as in our previous work on one-nucleon knockout reactions [4,6-8,5]. The three nuclei studied, belonging to a region of current interest [7,9-11], were produced in fragmentation of a $140 \mathrm{MeV} /$ nucleon ${ }^{40} \mathrm{Ar}$ beam from the Coupled-Cyclotron Facility at the NSCL. The secondary beams were selected in the A1900 fragment separator [12] with the momentum acceptance reduced to $0.5 \%$ in order to permit operation of the high-resolution S800 spectrograph [13] in dispersion-

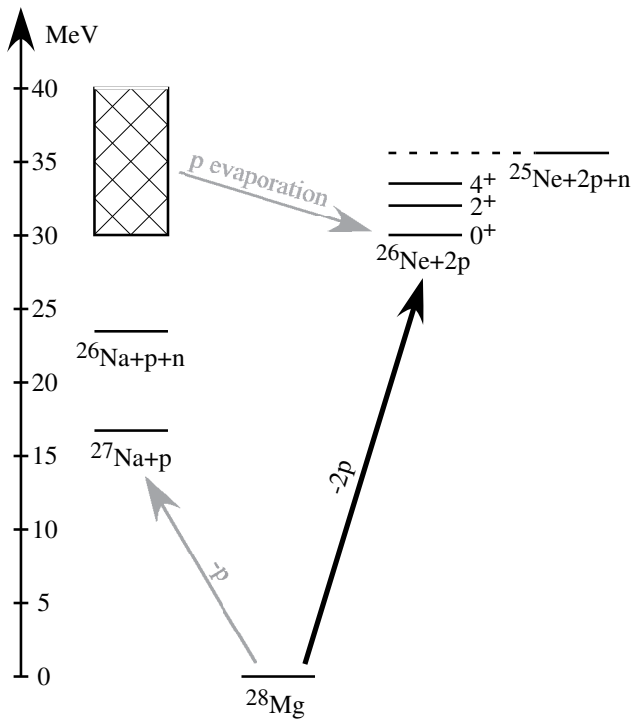

Figure 1: Diagram showing the possible two-proton removal reaction paths in the case of ${ }^{28} \mathrm{Mg}$.

matched mode. The reaction residues were momentum analyzed in the spectrograph and unambiguously identified by the energy loss and time of flight between the beam-monitoring scintillators located in the focal plane of the A1900 and scintillators in the focal plane of the S800 spectrograph.

The results from the measurements of the residues are shown in Fig. 2. Only a part of the parallel-momentum distribution could be detected in a single setting of the spectrograph, and the complete distribution shown for ${ }^{28} \mathrm{Mg}$ is a composite from three field settings. The inclusive cross sections given in the figures have been corrected for angular and momentum acceptance [13]. The secondary target was surrounded by the SeGA (Segmented Germanium Array) consisting of fourteen 32-fold segmented germanium detectors [14] arranged at a distance of $20 \mathrm{~cm}$ from the target in two rings with angles of $90^{\circ}$ and $37^{\circ}$ relative to the beam axis. The segmentation allowed for an event-by-event Doppler reconstruction of the energies of the $\gamma$ rays in coincidence with the knockout residues. Pseudo data from GEANT 3 simulations [15] successfully modeled the line shapes obtained after retroconversion to the center-of-mass system. The full-energy peak efficiencies obtained this way ranged from $3.2 \%$ at $0.885 \mathrm{MeV}$ to $1.8 \%$ at $2.02 \mathrm{MeV}$. The resolution of approximately $2.5 \%$ was limited by the close distance of the detectors, by the differential energy loss between projectile and fragment, which differ in $\mathrm{Z}$ by two units, and by the size of the target. The main states identified in Fig. 3 are all known from previous experiments, the $2^{+}$states from Coulomb excitation, see Ref. [9] and references therein, and the $4^{+}$ states from in-beam gamma spectroscopy $[16,17]$. For the detailed analysis of the ${ }^{28} \mathrm{Mg}$ results it is important that a $10 \%$ excess of intensity near $1.7 \mathrm{MeV}$ must be due to the second $2^{+}$and $0^{+}$states, decaying by gamma rays of 1.66 and $1.80 \mathrm{MeV}$ [16] and seen also in beta decay [18]. The reaction of ${ }^{34} \mathrm{Si}$ shows an apparent excess of gamma intensity above the adjusted continuum curve for energies above $1.4 \mathrm{MeV}$. This suggests that the cross section is fragmented to unobserved higher states and that it is not safe to deduce cross sections from an intensity balance. For ${ }^{30} \mathrm{Mg}$ (not shown in the figure) the statistics 


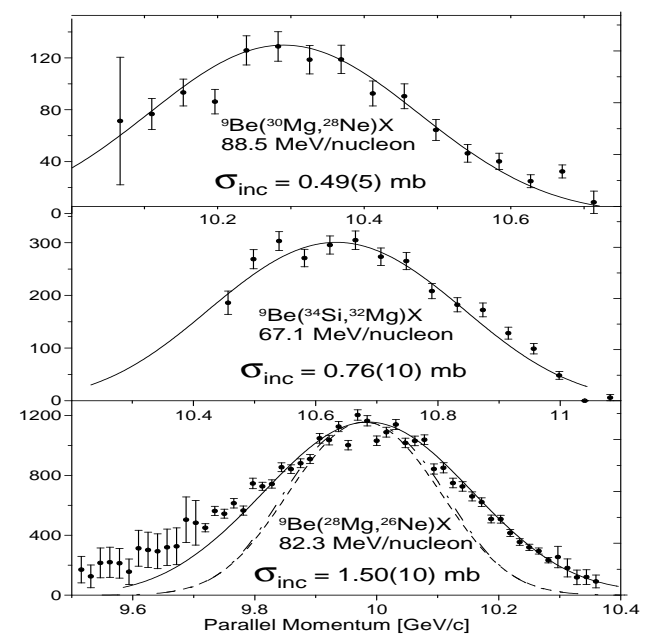

Figure 2. Parallel-momentum distributions for two-proton knockout reactions. The theoretical curves include the Lorentz correction and the broadening arising mainly from the target thickness. The full drawn curves are estimates for knockout of two protons in $0 d$ states. For ${ }^{28} \mathrm{Mg}$ the width (FWHM) without the stopping power correction would be $380 \mathrm{MeV} / \mathrm{c}$; the comparison value for two protons in $1 s$ states (dashed) is $282 \mathrm{MeV} / \mathrm{c}$ and for a single $0 d$ knockout (dashdotted) $298 \mathrm{MeV} / \mathrm{c}$.

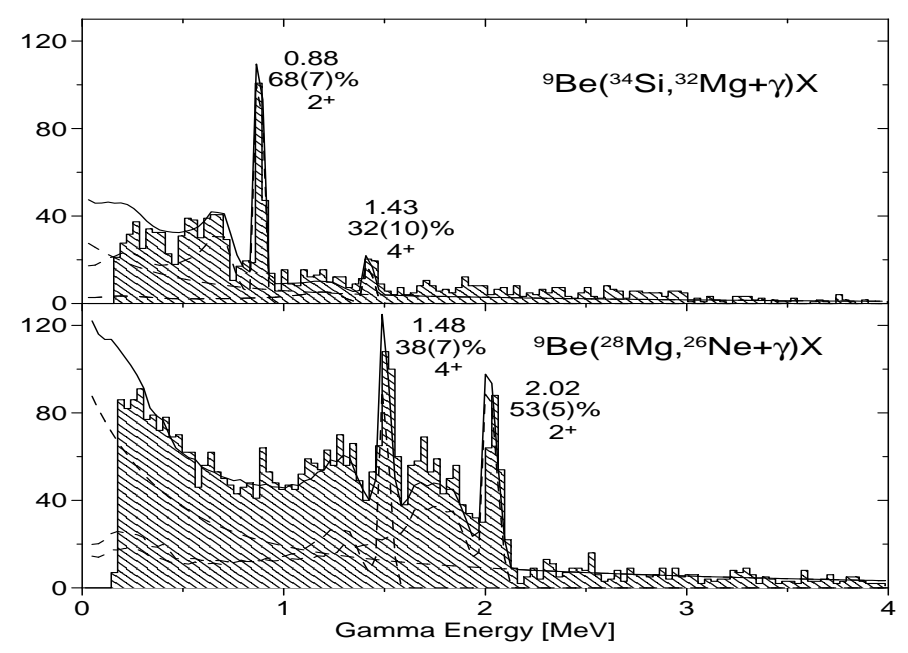

Figure 3. Gamma-ray spectra in counts per 32 $\mathrm{keV}$ bins. The main peaks, all representing $2^{+} \rightarrow$ $0^{+}$and $4^{+} \rightarrow 2^{+}$transitions, are labelled by the energy in $\mathrm{MeV}$ (uncertainty $0.02 \mathrm{MeV}$ ) and by the absolute intensity relative to the number of observed fragments. The dashed peak shapes are simulated response curves normalized to match the number of counts in the full-energy peaks. The continuous distribution (dashed line) is attributed to radiations from the target and is adjusted to the ${ }^{28} \mathrm{Mg}$ data as a sum of a straight line and an exponential. The same continuum, scaled with the number of residues, is shown in the ${ }^{34} \mathrm{Si}$ spectrum. The full-drawn lines represent the sum of all three components.

was low; the two main peaks were $1.32 \mathrm{MeV}\left(2^{+}\right), 53(13) \%$ and $1.73 \mathrm{MeV}\left(4^{+}\right), 39(13) \%$. In the following we restrict the analysis of the partial cross sections to the case of ${ }^{28} \mathrm{Mg}$, for which the gamma information appears close to complete. This is also the case that is simplest from a theoretical viewpoint since both the the initial and final states have a spherical configuration stabilized by the pronounced sub-shell closure at N=16 [10]. The energies agree well with a calculation using the $s d$ model space with the USD effective interaction $[19,20]$, see Table 1.

\section{A simple model}

A comprehensive theory for direct two-proton knockout will necessarily be more complex than that for knockout of a single nucleon. In the latter case the partial cross section for a given $j$ channel factorizes into a part describing the contribution from many-body nuclear structure ("the spectroscopic factor") and a part describing the reaction dynamics 
("the single-particle cross section") [5]. This no longer holds for the two-nucleon process. For this, many two-particle components may, within each total-angular-momentum channel, contribute coherently. The transition amplitude can then have significant interference effects reflecting combined contributions from structure and reaction geometry.

As such a theory does not yet exists, the calculation of the absolute cross section was performed using eikonal reaction theory assuming two uncorrelated protons. For a process engaging any two particles in the valence shell it is convenient to assume that the two particles are uncorrelated. Eikonal reaction theory [5] then gives the basic unit of cross section as (neglecting spin-orbit splitting)

\begin{tabular}{cccc|cccc}
\hline \hline$I^{\pi}$ & $p=2$ & 4 & 6 & $E_{\mathrm{th}}$ & $E_{\mathrm{ex}}$ & $S_{\mathrm{rel}}$ & $S_{\mathrm{ex}}$ \\
\hline $0^{+}$ & 1 & 1.33 & 1 & 0.00 & 0.00 & 1.6 & $2.4(5)$ \\
$2^{+}$ & 0 & 1.67 & 5 & 2.01 & 2.02 & 0.14 & $0.3(5)$ \\
$4^{+}$ & 0 & 3 & 9 & 3.66 & 3.50 & $(2.0)$ & $2.0(3)$ \\
$2^{+}$ & - & - & - & 3.45 & 3.7 & 0.46 & $0.5(3)$ \\
\hline \hline
\end{tabular}

Table 1: Theoretical spectroscopic factors $S_{p}\left(I^{\pi}\right)$ in the $d_{5 / 2}^{p}$ single sub-shell model (left) and comparison (right) of the experimental values with an $s d$ shell-model calculation for the case of ${ }^{28} \mathrm{Mg}$ (see [1]). $S_{\mathrm{ex}}$ is in units of $\sigma_{22}=0.29 \mathrm{mb}$.

$\sigma_{\ell_{1} \ell_{2}}=\int d \vec{b}\left|\mathcal{S}_{c}\right|^{2} \prod_{i=1,2} \frac{1}{2 \ell_{i}+1} \sum_{m_{i}}\left\langle\ell_{i} m_{i}\left|\left(1-\left|\mathcal{S}_{p i}\right|^{2}\right)\right| \ell_{i} m_{i}\right\rangle$

Here $\mathcal{S}_{\mathrm{c}}$ and the $\mathcal{S}_{p}$ are the elastic S-matrices (profile functions) for the core- and protontarget systems, functions of their individual impact parameters, and calculated in the optical limit of Glauber theory. The individual proton-core relative motion wave functions $|\ell m\rangle$ are calculated in Woods-Saxon potentials with depths adjusted to reproduce the proton separation energies. We neglect recoil of the heavy core. Equation (1) allows a simple interpretation as the integral over the two-dimensional impact parameter and average over $m$ sub-states of the joint probability of the core being elastically scattered and of the two protons being absorbed. Diffractive breakup processes are assumed to be negligible for these very deeply bound protons. (For the simpler case of diffractive breakup of a single proton a sum-rule estimate gives an upper limit of $20 \%$; the contribution is most likely smaller in the two-proton case.) For ${ }^{28} \mathrm{Mg}$ the cross section $\sigma_{22}$ is $0.29 \mathrm{mb}$, $\sigma_{00}$ is $0.35 \mathrm{mb}$, and $\sigma_{20}$ is $0.32 \mathrm{mb}$. The corresponding $\ell=2(0)$ single-particle (unit) cross sections for knockout of one proton are 11.6 (14.8) $\mathrm{mb}$. For ${ }^{30} \mathrm{Mg} \sigma_{22}$ is $0.22 \mathrm{mb}$, and for ${ }^{34} \mathrm{Si} \sigma_{22}$ is $0.144 \mathrm{mb}$.

In order to obtain reaction cross sections from the unit values we need the equivalent of a spectroscopic factor. From the approximation of no correlations it follows from simple combinatorics that for $p$ particles in the valence shell this factor is $S_{p}=p(p-1) / 2$. Thus for ${ }^{28} \mathrm{Mg}$ with $p=4$ we obtain $6 \cdot \sigma_{22}=1.8 \mathrm{mb}$, measured value $1.50(10) \mathrm{mb}$, for ${ }^{34} \mathrm{Si}$ with $p=6$ we obtain $2.2 \mathrm{mb}$, measured value $0.76(10) \mathrm{mb}$, and for ${ }^{30} \mathrm{Mg}$ with $p=4$ we obtain $1.3 \mathrm{mb}$, measured value $0.49(5) \mathrm{mb}$. In view of the approximations made, all three cases must be considered to show good agreement between experiment and the direct-reaction model, demonstrating that we have, at least, a semi-quantitative understanding of the absolute cross sections. Assuming a single active $j$ shell, we can calculate spectroscopic factors to individual excited levels. For the even- $Z$ elements neon to silicon it is a good first approximation to assume that all valence protons numbering $p=2,4,6$ are in the $0 d_{5 / 2}$ subshell. The only possible final states now have $I=0,2,4^{+}$. The corresponding spectroscopic factors $S_{p}\left(I^{\pi}\right)$ are given in Table 1 . The values for $p=4$ are in qualitative 
agreement with results for ${ }^{28} \mathrm{Mg}$, obtained by dividing the partial cross sections obtained from an input-output intensity balance by the unit cross section of Eq. (1). However, the low cross section to the $2^{+}$state can only be reproduced after a full diagonalization in the sd shell (see [1]).

\section{Conclusion}

We have shown that when a neutron-rich projectile reacts with a light nuclear target, the knockout of two protons occurs as a direct reaction. Consequently, the observed partial cross sections to individual final levels convey selective information about nuclear structure. We expect the same to be the case for two-neutron knockout from a proton-rich nucleus. More experiments and also the development of a more complete reaction theory are interesting challenges. The experimental results suggest that the pattern and absolute magnitudes of the partial cross sections will provide specific information on the detailed nature of the states involved. It is interesting to note that, measured from beta stability $\left({ }^{31} \mathrm{P}\right),{ }^{28} \mathrm{Mg}$ is less than half way to the drip line $\left({ }^{22} \mathrm{C}\right)$. This implies that this new direct reaction will be a viable method in a wide region extending to the drip line.

\section{REFERENCES}

1. D. Bazin et al. Phys. Rev. Lett. 91, 012501 (2003).

2. G.R. Satchler, Direct Nuclear Reactions (University Press, Oxford, 1983) pp. 1-833.

3. H. Feshbach, Theoretical Nuclear Physics: Nuclear Reactions (Wiley, New York, 1992), p. 455.

4. A. Navin et al. Phys. Rev. Lett. 81, 5089 (1998).

5. J.A. Tostevin, J. Phys. G 25, 735 (1999).

6. V. Maddalena et al., Phys. Rev. C 63, 024613 (2001).

7. J. Enders et al., Phys. Rev. C 65, 034318 (2002)

8. P.G. Hansen and B.M. Sherrill, Nucl. Phys. A 693, 133 (2001).

9. B. V. Pritychenko et al., Phys. Lett. B 461, 322 (1999); 467, 309 (1999) (erratum).

10. T. Otsuka et al., Phys. Rev. Lett. 87, 082502 (2001).

11. B.A. Brown, Prog. Part. Nucl. Phys. 47, 517 (2001).

12. D. J. Morrissey et al., Nucl. Instrum. Methods in Phys. Res., Sect. B 204, 90 (2003).

13. D. Bazin et al., Nucl. Instrum. Methods in Phys. Res., Sect. B 204, 629 (2003).

14. W. F. Mueller et al., Nucl. Instrum. Meth. A 466, 492 (2001).

15. GEANT-detector description and simulation tool, version 3.21, CERN Program Library Long Writeup W5013, 1994.

16. O. Sorlin et al., Nucl. Phys. A 685, 186c (2001).

17. F. Azaiez et al., Eur. Phys. J. A 15, 93 (2002).

18. A.T. Reed et al., Phys. Rev. C 60, 024311 (1999)

19. B. A. Brown and B.H. Wildenthal, Ann. Rev. Nucl. Part. Sci. 38, 29 (1988); B. H. Wildenthal, Prog. Part. Nucl. Phys. 11, 5 (1984).

20. B. A. Brown, http://www.nscl.msu.edu/ brown/sde.htm 